\title{
Team-Based Hypertension Management in Outpatient Settings
}

\author{
Shawn R. Smith, PharmD, Emmanuelle Schwartzman, PharmD, and Micah Hata, PharmD
}

\section{ABSTRACT}

Objective: To review the current literature regarding the clinical effectiveness and cost-effectiveness of implementing hypertension team-based care (TBC) interventions in the outpatient setting, and discuss challenges to implementation.

Methods: A literature review was conducted of metaanalyses, systematic reviews, and randomized controlled trials comparing TBC models to usual care for hypertension management.

Results: Compared to usual care, TBC models have demonstrated greater blood pressure reductions and improved blood pressure control rates. Evidence was strongest for models involving nurses and pharmacists whose roles included medication management, patient education and counseling, coordination of care and follow-up, population health management, and performance measurement with quality improvement. Although TBC results in an increase in health care costs, the overall long-term benefits support the costeffectiveness of these models over usual care. The most common barriers to TBC implementation include underutilization of technology, stakeholder engagement, and reimbursement issues.

Conclusion: Hypertension TBC models have been shown to be clinically effective and cost-effective, but continued research comparing different models is warranted to determine which combination of health professionals and interventions is most impactful and cost-effective in practice. An implementation science approach, in which TBC models unique to each organization's situation are created, will be useful to identify and overcome challenges and provide a solid foundation for sustainment.

Keywords: blood pressure; pharmacist; nurse; nurse practitioner; cost-effectiveness; team-based care.
A pproximately 1 in 3 US adults-or about 100 million people-have high blood pressure, and only about half (48\%) have their blood pressure under control. ${ }^{1}$ Effective blood pressure management has been shown to decrease the incidence of stroke, heart attack, and heart failure. ${ }^{2-4}$ The American College of Cardiology/American Heart Association (ACC/ AHA) 2017 blood pressure guidelines recommended lower thresholds for diagnosing hypertension and initiating antihypertensive medication, and intensified the blood pressure goal to less than $130 / 80 \mathrm{~mm} \mathrm{Hg} .^{5}$ Changing practice standards to more intensive blood pressure goals requires significant adjustments by clinicians and health care systems. In fact, new guideline uptake is often delayed, ignored, or sparsely applied. ${ }^{6}$ Due to this dramatic change in hypertension practice standards, the ACC/AHA guidelines support interdisciplinary team-based care (TBC) for hypertension management. ${ }^{5,7}$ Additionally, the Centers for Disease Control and Prevention (CDC) and the Community Preventive Services Task Force (CPSTF) promote TBC to improve blood pressure control in their initiatives to prevent heart disease and stroke. ${ }^{8,9}$

The National Academy of Medicine defines TBC as "the provision of health services to individuals, families, and/or their communities by at least 2 healthcare providers who work collaboratively with patients and their caregivers - to the extent preferred by each patient-to accomplish shared goals within and across settings to achieve coordinated, high-quality care."10 Specific goals for TBC in hypertension treatment are listed in Table $\mathbf{1}$, and a checklist of key elements of TBC to consider before implementation are presented in Table 2.

From Western University of Health Sciences College of Pharmacy, Department of Pharmacy Practice and Administration, Pomona, CA. 
Table 1. Goals of Team-Based Care of Patients

With Hypertension

- Improve clinical workflow

- Enhance patient education

- Provide closer blood pressure follow-up

- Facilitate medication titration

- Facilitate laboratory follow-up

- Improve adherence

- Reduce clinician burn-out

Adapted from 2019 AHAACC Clinical Performance and Quality Measures for Adults With High Blood Pressure. ${ }^{69}$

TBC has been shown to have many advantages, including increased access to care due to expanded hours of operation and shorter wait times. ${ }^{11}$ Team-based models also provide effective and efficient delivery of patient education, behavioral health care, and care coordination. ${ }^{12-14}$ Patients are more likely to receive high-quality care when multiple providers, each with varied expertise, are on the health care team. ${ }^{11,15}$ Furthermore, clinicians report improved professional job satisfaction related to their ability to practice in environments where they are encouraged to work at the top of their licenses. ${ }^{16}$ Consequently, TBC has been accepted as a vital part of the patient-centered medical home $(\mathrm{PCMH})$ model. ${ }^{17-19}$ Standards set by the National Committee for Quality Assurance (NCQA) include TBC as a requirement health systems must meet in order to achieve the highest level of PCMH recognition. While a team-based approach offers substantial benefits and is recognized as a marker of quality, implementation has presented various challenges, and the sustainability of these models in care settings has been questioned. ${ }^{20}$ In this article, we review the current literature regarding the clinical effectiveness and cost-effectiveness of implementing hypertension TBC interventions in the outpatient setting. We also discuss the challenges and opportunities of implementing this strategy in health systems and community settings in the United States.

\section{Evidence of Impact and Effectiveness}

Various models of hypertension TBC have been shown to increase the proportion of individuals with controlled blood pressure and to lead to a reduction in both systolic (SBP) and diastolic blood pressure (DBP), resulting in a strong recommendation for TBC approaches by the 2017 ACC/AHA blood pressure guidelines.5,21-25 There is great diversity in the types of hypertension treatment models studied, with few utilizing physician specialists and most utilizing nonphysician providers, such as community health workers, physician assistants, nurses, nurse practitioners, dietitians, social workers, and pharmacists. ${ }^{22,26-29}$ These professionals share duties of hypertension management with primary care physicians to reduce the burden of responsibility for care on any single provider type. TBC is patient-centered, and typically includes interprofessional collaboration, treatment algorithms, adherence counseling, frequent follow-up, home blood pressure monitoring, and patient selfmanagement education.

Numerous studies have supported implementation of TBC in recent years. A systematic review and metaanalysis of 100 trials of hypertension TBC involving 55,920 patients concluded that the most effective blood pressure-lowering strategies use multilevel, multicomponent approaches to address barriers to hypertension control. Nonphysician providers are often involved in measuring blood pressure, ordering and assessing laboratory tests, and titrating medications..$^{30}$ Compared with usual care, TBC with physician medication titration resulted in reductions in mean SBP and DBP $(6.2 \mathrm{~mm} \mathrm{Hg}$ and $2.7 \mathrm{~mm} \mathrm{Hg}$, respectively), while $\mathrm{TBC}$ with nonphysician medication titration also resulted in reductions in mean SBP and DBP (7.1 $\mathrm{mm} \mathrm{Hg}$ and $3.1 \mathrm{~mm} \mathrm{Hg}$, respectively).

Nurses and pharmacists are specifically mentioned by the 2017 ACC/AHA blood pressure guidelines as essential members of the hypertension treatment team. ${ }^{5}$ Randomized controlled trials (RCTs) and metaanalyses of TBC involving nurse or pharmacist interventions demonstrated greater reductions in SBP and/or greater attainment of blood pressure goals compared to usual care. ${ }^{21,26,31,32}$ The literature supports the roles of nurses and pharmacists in hypertension management in all aspects of care, including medication management, patient education and counseling, coordination of care and follow-up, population health management, and performance measurement with quality improvement. ${ }^{33}$ 


\section{Nurses}

Nurses are commonly part of TBC hypertension management programs. One meta-analysis and systematic review of international RCTs compared nurse, nurse prescriber (United Kingdom), and nurse practitioner interventions for hypertension with usual care. Interventions that included a stepped treatment algorithm and nurse prescribing showed greater reductions in SBP $(8.2 \mathrm{~mm}$ $\mathrm{Hg}$ and $8.9 \mathrm{~mm} \mathrm{Hg}$, respectively) compared to usual care. ${ }^{31}$ Similarly, models that utilized telephone monitoring demonstrated greater achievement of blood pressure targets, while those that involved home monitoring showed significant reductions in blood pressure. Another international meta-analysis and systematic review of 11 nurseled interventions in hypertensive patients with diabetes demonstrated a $5.8 \mathrm{~mm} \mathrm{Hg}$ mean decrease in SBP compared to physician-led care. However, nurse-led care was not superior in achievement of study targets. ${ }^{34}$

A recent meta-analysis and systematic review, performed by Shaw and colleagues, sought to determine whether nurse-led protocols are effective for outpatient management of adults with diabetes, hypertension, and hyperlipidemia. All of the included studies involved a registered nurse who titrated medications by following a protocol, and most were RCTs comparing the nurse protocols to usual care. Overall, mean SBP and DBP decreased by $3.86 \mathrm{~mm} \mathrm{Hg}$ and $1.56 \mathrm{~mm} \mathrm{Hg}$, respectively, while blood glucose and lipid levels were also reduced compared to usual care. ${ }^{24}$

Limited RCT data have been published since the Shaw et al meta-analysis. A single-blind RCT was performed in an urban community health care center in China among patients with uncontrolled blood pressure (SBP $\geq 140$ $\mathrm{mm} \mathrm{Hg}$ and/or DBP $\geq 90 \mathrm{~mm} \mathrm{Hg}$ ). ${ }^{35}$ The study group received care via a nurse-led model, which included a delivery design system, decision support, clinical information system, and self-management support, and the control group received usual care. At 12 weeks, patients in the study group had significantly lower blood pressure than control patients, with mean SBP/DBP reduction of 14.37/7.43 $\mathrm{mm} \mathrm{Hg}$ and 5.10/2.69 $\mathrm{mm} \mathrm{Hg}$, respectively $(P<0.01)$. Improved medication adherence and increased patient satisfaction were other benefits of the nurse-led model.

\section{Table 2. Checklist for Team-Based Hypertension Care}

Team members include a lead clinician, clinical support (ie, pharmacy, nurse, physician assistant), administrative support, and an expert for referral. Team meetings should occur at least quarterly to evaluate delivery of care for patients with hypertension.

\section{Program Elements}

1. Patient education materials or sessions on hypertension

2. Availability of blood pressure-specific follow-up in 1 month (telephone based, with home blood pressure monitoring, telehealth, or clinical support or clinician follow-up)

3. Ability of patients to contact team member in a timely fashion about hypertension concerns (telephone, secure electronic health record messaging, email, urgent appointments)

4. Algorithm for medication titration led by clinical support team member and lead clinician supervisor

5. Timely follow-up and monitoring of laboratory results, with titration by relevant drug class

6. Monitoring of adherence by using pharmacy fill data

7. Provider-specific performance reports with hypertension metrics

Adapted from 2019 AHA/ACC Clinical Performance and Quality Measures for Adults With High Blood Pressure. ${ }^{69}$

Nurse case managers (NCM) also play a critical role in hypertension management, coordinating health care services to meet patient health needs. Ogedegbe sought to evaluate the comparative effectiveness of home blood pressure telemonitoring (HBPTM)+NCM versus HBPTM alone on SBP reduction in black and Hispanic stroke survivors. ${ }^{36,37}$ NCMs evaluated patient profiles, counseled patients on target lifestyle behaviors, and reviewed home blood pressure data. At 6 months, SBP declined by $13.63 \mathrm{~mm} \mathrm{Hg}$ from baseline in the HBPTM+NCM group and $6.31 \mathrm{~mm} \mathrm{Hg}$ in the HBPTM alone group $(P<0.0001)$. At 12 months, SBP in the HBPTM+NCM group declined by $14.76 \mathrm{~mm} \mathrm{Hg}$, while blood pressure in the HBPTM alone group declined by $5.53 \mathrm{~mm} \mathrm{Hg}(P<0.0001)$.

\section{Pharmacists}

Clinical pharmacists are also widely utilized in TBC models for hypertension management. Typical models involve pharmacists entering into collaborative practice 
agreements with physicians, leading to optimization of medications, avoidance of adverse drug events, and transitional care activities focusing on medication reconciliation and patient education in outpatient settings..$^{30,38}$ The largest and most recent meta-analysis of pharmacist interventions, conducted in 2014 by Santschi et al, ${ }^{23}$ combined 2 previous systematic reviews to include a total of 39 RCTs with 14,224 patients. ${ }^{32,39}$ Pharmacist interventions included patient education, recommendations to physicians, and medication management. Compared with usual care, pharmacist interventions showed greater reductions in SBP $(7.6 \mathrm{~mm} \mathrm{Hg})$ and DBP $(3.9 \mathrm{~mm} \mathrm{Hg}){ }^{23}$

Numerous studies substantiating the impact of pharmacist interventions on clinical outcomes have heavily influenced clinical practice and guideline development. Carter et al conducted a prospective, multistate, cluster-randomized trial in 32 primary care clinics to evaluate whether clinics randomized to receive the pharmacist-physician collaborative care model (PPCCM) achieved better blood pressure outcomes versus clinics randomized to usual care. ${ }^{25}$ Investigators enrolled 625 patients with uncontrolled hypertension, $50 \%$ of whom had a prior diagnosis of diabetes mellitus or chronic kidney disease. The primary outcome of blood pressure control at 9 months in the intervention clinics compared to the control clinics was $43 \%$ and $34 \%$, respectively $(P=0.059)$. The difference in mean SBP/DBP between the intervention and control clinics for all patients at 9 months was $-6.1 /-2.9 \mathrm{~mm} \mathrm{Hg}$. In a post-hoc analysis of patients with chronic kidney disease and diabetes, the pharmacist-intervention group had a significantly greater mean SBP reduction and higher blood pressure control rates compared to usual care at 9 months. ${ }^{40}$

A pre-specified secondary analysis from the Carter et al study determined that, in patients from racial minority groups, the mean SBP was $7.3 \mathrm{~mm} \mathrm{Hg}$ lower in those who received the intervention compared to those in the control group $(P=0.0042)$. ${ }^{41}$ In patients with less than 12 years of education, those in the intervention group had a mean SBP $8.1 \mathrm{~mm} \mathrm{Hg}$ lower than the SBP of those in the control group ( $P=0.0001$ ). Similar reductions in blood pressure occurred in patients with low income, Medicaid beneficiaries, or those without insurance. This study demonstrated that pharmacist interventions reduced racial and socioeconomic disparities in blood pressure treatment.

Other studies of pharmacist interventions in underserved populations have yielded positive results. In a retrospective review of uninsured patients, blood pressure control rates in a pharmacist-driven primary care clinic ranked in the 90th percentile of NCQA benchmarks, and was superior to the 2013 reported mean for commercial insurers. ${ }^{42}$ Similarly, another retrospective cohort study of a PPCCM on time to goal blood pressure in uninsured patients with hypertension showed the median time to blood pressure goal was 36 days in the PPCCM cohort versus 259 days in usual care cohorts $(P<0.001) .{ }^{43} \mathrm{~A}$ post-hoc analysis revealed the mean time-in-therapeutic blood pressure range was $46.2 \% \pm 24.3 \%$ in the PPCCM group and $24.8 \% \pm 27.4 \%$ in the usual care group $(P<0.0001)$. The blood pressure control rates at 12 months were $89 \%$ in the PPCCM group compared with $50 \%$ in the usual care group $(P<0.0001){ }^{44}$

Tsuyuki et al conducted the RxACTION study, a multicenter RCT evaluating the effectiveness of enhanced pharmacist care versus usual care in 23 Canadian community pharmacies and outpatient clinics following a 6-month intervention. ${ }^{45}$ Enhanced pharmacy services included pharmacist assessment of and counseling about cardiovascular disease risk and blood pressure control, review of current antihypertensive medications, and prescribing/titrating drug therapy, as needed, through independent prescriptive authority. Compared to the usual care group $(n=67)$, the intervention group had a reduction in SBP of $6.6 \mathrm{~mm} \mathrm{Hg}(P=0.006)$ and in DBP of $3.2 \mathrm{~mm} \mathrm{Hg}(P=0.01)$. This study expanded the pharmacists' scope of practice, showing evidence for enhancing pharmacist roles on the hypertension care team. Tsuyuki et al also conducted the RxEACH randomized trial, which evaluated community pharmacist cardiovascular risk reduction interventions and showed an improvement in SBP and DBP, with reported results comparable to RXACTION. ${ }^{46}$

Victor et al conducted the landmark Black Barbershop Study, a cluster RCT involving 319 non-Hispanic black male patients with hypertension from 52 black-owned barbershops. ${ }^{47,48}$ Barbershops were assigned to 1 of 2 groups. The control group consisted of barbers who 
encouraged lifestyle modifications and made referrals to primary care providers. The intervention group had pharmacists who met regularly with participants at the barbershops and measured blood pressure, encouraged lifestyle changes, and prescribed drug therapy under collaborative practice agreements with physicians. Both groups demonstrated improvements in blood pressure outcomes, but the intervention group showed greater improvement in SBP and achievement of blood pressure goals compared to the control group. The results in the intervention group proved sustainable over the course of a year, even after the frequency of pharmacists' visits was reduced. At 6 months, the mean SBP fell by $27.0 \mathrm{~mm} \mathrm{Hg}$ (to $125.8 \mathrm{~mm} \mathrm{Hg}$ ) in the intervention group, as compared to a $9.3 \mathrm{~mm} \mathrm{Hg}$ (to $145.4 \mathrm{~mm} \mathrm{Hg}$ ) reduction in the control group $(P<0.001)$, and blood pressure less than $130 / 80 \mathrm{~mm} \mathrm{Hg}$ was achieved among $63.6 \%$ of the participants in the intervention group versus $11.7 \%$ in the control group $(P<0.001)$.

This community-level trial brought pharmacists to the barbershop and made them an essential part of the health care team through the endorsement of the barber, who the participants trusted and with whom they had a relationship. Long-standing issues related to distrust of the medical profession by this population were addressed, and trusted community barbershops were utilized as safe spaces for health care delivery. Health care professionals should consider utilizing community locations that other minority populations perceive as social centers and safe places, to reduce health disparities and barriers to care. However, models that bring care to patients need further economic and feasibility evaluations.

\section{Other Health Care Professionals and Future Studies}

In addition to models led by nurses and pharmacists, studies have also assessed models of TBC incorporating other health care professionals, including registered dietitians, medical assistants, community health workers, and health coaches (NCT02674464).49,50 Ongoing studies are also looking at the impact of TBC on underserved communities (NCT02674464, NCT03504124). Involving a variety of health care professionals with different communities and populations in TBC studies is warranted to determine the optimal settings in which to utilize different skill sets.

The Impress Study involves nurses who are assessing lifestyle risk and developing an action plan according to a standardized procedure, which may be advantageous given the degree of heterogeneity found in other TBC models. ${ }^{51}$ There are also studies underway or recently published that compare different components of TBC in order to determine which combination of TBC elements is preferred. Some of these have shown the benefits of using clinical decision-support systems (through a guideline-based treatment protocol) or training programs with ongoing support. ${ }^{52,53}$ Continued research comparing different TBC models is needed to determine which combination of health professionals and interventions is most impactful in practice.

\section{Health care professionals should consider utilizing community locations that other minority populations perceive as social centers and safe places, to reduce health disparities and barriers to care.}

\section{Cost-Effectiveness}

According to the CDC, TBC in hypertension management has proven to be cost-effective. ${ }^{54}$ Systematic reviews and meta-analyses assessing the cost-effectiveness of TBC in hypertension management have been conducted. ${ }^{26,27,29,55-58}$ While the general consensus supports this approach as being cost-effective, these determinations are based on studies that are widely heterogeneous. In each of these studies, different types of costs are taken into account when determining cost-effectiveness. The range of costs can be quite wide, depending on how they are calculated, making it difficult to determine the true cost-effectiveness of different TBC models.

Intervention cost is represented by the amount of money spent to implement and maintain the intervention beyond the cost of usual care or the cost without the intervention. For TBC, intervention cost consists of personnel resources such as provider time, patient 
time, and non-personnel resources, including rent and utilities. Studies show that intervention costs for TBC can range from $\$ 35$ to $\$ 1350$ per person per year (mean, \$618; median, \$428). ${ }^{27,56}$ One analysis, based on 20 studies comparing TBC to usual care, calculated an intervention cost of $\$ 284$ per person per year, ${ }^{55}$ while another study showed an intervention cost of $\$ 525$ per enrollee per year. ${ }^{56}$ Intervention cost can vary by the type of provider that is used, the amount of time spent per patient, and the setting where services are provided. Overall, the intervention cost of implementing TBC for hypertension management is consistently higher than the cost of usual care.

Health care cost is another factor to consider. It is the difference in the cost of health care products and services that are utilized in the process of TBC, as compared to care that is provided in the absence of TBC. Health care costs include the costs associated with hospitalizations, outpatient visits, emergency room visits, and medications. One study estimated a median health care cost of hypertension TBC of $\$ 65$ per person per year. ${ }^{55}$ Overall, studies evaluating the impact of TBC for hypertension management on health care costs were mixed, with some showing that TBC resulted in an increase in health care cost, and others showing a savings compared to usual care..$^{58}$ The variability in health care costs was due to the different number of health care components and comorbidities of the patients included in the studies. Also, study duration affected the estimated health care costs of TBC. Most studies did not assess long-term health care cost savings that could be achieved from prolonged blood pressure control. ${ }^{58}$ When considering both intervention and health care cost, Jacob et al estimated that TBC increased overall net cost by a median value of $\$ 329$ per person per year. ${ }^{55}$ While some studies did attribute an overall reduction in health care costs to TBC for hypertension management, on average, teambased models increased health care costs compared to usual care. ${ }^{27,29,55,58,59}$

However, health care costs do not take into account the long-term reductions in morbidity and mortality or increased quality-adjusted life years (QALY) that result from improved blood pressure control attributed to TBC. In most cost-effectiveness studies, an intervention is considered to be cost-effective if the cost per QALY gained is less than the accepted threshold of $\$ 50,000 .{ }^{55}$ One study estimated that the cost per QALY of TBC in hypertension management is $\$ 4763,,^{55,60}$ while another study estimated a median cost per QALY of $\$ 9716$ to $\$ 13,992 .{ }^{55}$ A systematic review of 34 international studies estimated the median cost per QALY to be $\$ 13,986$, ranging from $\$ 6683$ to $\$ 58,610.57$ The wide range in cost can be attributed to the variability in interventions, health outcomes used to measure effectiveness, and the settings and countries where the studies were conducted. In another study, a TBC intervention involving pharmacists resulted in a cost per QALY of $\$ 26,800 .^{61}$ The intervention was found to be cost-effective for higher-risk patients, defined as those having diabetes, a smoking history, dyslipidemia, or obesity. For patients who did not have these risk factors, the cost per QALY increased to $\$ 43,330 .{ }^{61}$ Thus, the patient population should be considered before implementing a TBC model. Furthermore, the increased use of technology, allowing for more efficient provision of services and communication between providers, could reduce intervention costs and lead to increased cost efficacy in these models.

The variation in the models used for TBC makes it difficult to draw conclusions on the cost-effectiveness of these interventions. Although it is apparent that TBC in general is cost-effective, more studies are needed comparing different team-based models to determine which specific ones are most cost-effective.

\section{Challenges to Implementation of Team-Based Care}

Recognizing and addressing the challenges inherent to a TBC approach is important to the sustainability of such a model within various settings and institutions. Numerous studies conducted on team-based models have identified common challenges that appear to be consistent across multiple settings. These challenges can be categorized as financial, provider-specific, and technology.

\section{Financial Barriers}

Although studies have demonstrated the cost-effectiveness of controlling hypertension and preventing serious complications, health systems are still confronted with the 
challenge of covering the cost for TBC implementation and maintenance. ${ }^{29}$ The 2 main financial barriers for TBC services are stakeholder engagement and reimbursement for services. According to Kennelty et al, stakeholder engagement is key to the sustainability of the service. ${ }^{27}$ However, decisions by stakeholders on cost are influenced by many factors, which include available funds, perceived value, and estimates for return on investment. Additionally, interventions must align with the organization's mission and vision and be feasible to implement, and organizations must have the capacity for administrative support. ${ }^{29}$ These various financial decisions may greatly influence the sustainability of a TBC model.

The reimbursement challenges for individual providers are an additional barrier to the sustainability of the service. In the United States, most providers are reimbursed via fee-for-service payment plans, but these plans do not reimburse all clinical providers because they are not all recognized as licensed providers. ${ }^{62,63}$ For example, pharmacists are not recognized by the Centers for Medicare \& Medicaid Services as licensed health care providers, which limits their ability to be reimbursed for clinical services provided outside of a traditional dispensing role. Furthermore, state laws determine the services nonphysician providers can offer and how they are recognized for reimbursement by tertiary payers. For instance, pharmacist roles, such as ordering labs and modifying or prescribing medication regimens, vary greatly between states.,73,64 Financial barriers are a major challenge facing the sustainability of a TBC hypertension service, so including all stakeholders in the decision-making process may improve the organization's ability to sustain the service.

\section{Provider-Specific Barriers}

Notable barriers that are attributed to providers include lack of knowledge, lack of time, lack of initiative to change blood pressure medications, and inability to reach intensive blood pressure goals set in guidelines. ${ }^{29}$ Studies such as the SPRINT trial have significantly impacted clinical guideline cut-offs for blood pressure, but reaching the intensive blood pressure goals from clinical trials is difficult to emulate in clinical practice. ${ }^{65}$ In a typical clinical setting, providers may lack the confidence to make adjustments in therapy based on a single blood pressure measurement, and clinical inertia, defined as failure of health care providers to modify therapy when indicated, ${ }^{66}$ may contribute to the inability to achieve blood pressure goals. Many factors contribute to clinical inertia, including lack of knowledge, time, or clinical protocols on how to modify therapy, causing providers to delay clinical decisions. Implementing site-specific protocols and utilizing hypertension specialist health care professionals in TBC can address the barriers contributing to clinical inertia.

Although it is apparent that TBC in general is cost-effective, more studies are needed comparing different teambased models to determine which specific ones are most cost-effective.

\section{Technology Barriers}

A common barrier in a variety of services, but especially prevalent in a TBC service, is access to an electronic health record (EHR) for all providers treating the patient. Some providers who are not directly tied to the same clinical site as the patient's primary care provider may not have adequate access to the full EHR. For example, pharmacists who are managing hypertension in a TBC model in a community pharmacy may have access only to health information from prescription records. Patient interviews may not provide the pharmacist with adequate information about laboratory results, vitals, and other medical information and history for the patient, making it difficult for the pharmacist to make a proper recommendation for treatment. ${ }^{27}$ Depending on the setting, communication between providers may be a barrier in achieving optimal outcomes, especially when providers do not have access to a shared medical record.

In addition, patients often lack access to technology used to manage hypertension. Many new technologies exist that aid patients in managing their blood pressure, such as smart phone applications to track blood pressure readings and alarms to remind patients to take their med- 
ications. Studies have shown that telemonitoring of blood pressure measurements and management of hypertension, especially in combination with TBC, is effective and reduces costs compared to usual care. ${ }^{67}$ However, the lack of equal access to the various technologies available may inhibit the success of a TBC hypertension program. Patients may lack access, knowledge, or financial means to utilize the various methods available for managing their hypertension electronically. ${ }^{29}$

\section{Conclusion}

Incorporating nonphysician providers into the health care team for the treatment of hypertension has proven to be more effective than usual care and has been recognized by recent guidelines as a best practice approach to achieving blood pressure goals. Multiple studies have demonstrated that TBC utilizing nurses and pharmacists can improve blood pressure management. While adding members to the team increases health care costs, the long-term benefits of achieving optimal blood pressure goals contribute to the overall cost-effectiveness of TBC strategies over usual care. However, comparisons between different TBC models are warranted to determine which combination of health care professionals and/or interventions is most effective. Cost-analysis estimates are difficult to compare due to widely varied methodology and variance in the models that have been employed. Studies must consider pathways to overcoming reimbursement issues, provider-specific challenges, and technology barriers. Follow-up and monitoring after initiation of drug therapy for hypertension control should include systematic strategies to help improve blood pressure, including use of home blood pressure monitoring, TBC, and telehealth strategies. Future implementation science approaches to hypertension TBC models within specific clinic settings will be useful to identify and overcome challenges and will help to determine the populations who will benefit most, allowing for greater success in sustaining TBC models.

Corresponding author: Shawn R. Smith, PharmD, 309 E. 2nd Street, Pomona, CA 91766; shawnsmith@westernu.edu.

Financial disclosures: None.

\section{References}

1. Fryar CD, Ostchega $Y$, Hales CM, et al. Hypertension prevalence and control among adults: United States, 2015-2016. NCHS Data Brief. 2017(289):1-8.

2. Ambrosius WT, Sink KM, Foy CG, et al. The design and rationale of a multicenter clinical trial comparing two strategies for control of systolic blood pressure: The Systolic Blood Pressure Intervention Trial (SPRINT). Clin Trials. 2014;11:532-546.

3. Lawes CM, Bennett DA, Feigin VL, Rodgers A. Blood pressure and stroke: an overview of published reviews. Stroke. 2017;35:776-785.

4. Zanchetti A, Thomopoulos C, Parati G. Randomized controlled trials of blood pressure lowering in hypertension: A critical reappraisal. Circ Res. 2015;116:1058-1073.

5. Whelton PK, Carey RM, Aronow WS, et al. 2017 ACC/AHA AAPA/ABC/ACPM/ AGS/APhA/ASH/ASPC/NMA/PCNA guideline for the prevention, detection, evaluation, and management of high blood pressure in adults: a report of the American College of Cardiology/American Heart Association Task Force on Clinical Practice Guidelines. J Am Coll Cardiol. 2018;71:e127-e248.

6. Grol R. Successes and failures in the implementation of evidence-based guidelines for clinical practice. Med Care. 2001; 39:|146- 1154.

7. Brush JE, Handberg EM, Biga C, et al. 2015 ACC health policy statement on cardiovascular team-based care and the role of advanced practice providers. J Am Coll Cardiol. 2015;65:2118-2136.

8. Centers for Disease Control and Prevention. Best practices for cardiovascular disease prevention programs: a guide to effective health care system interventions and community programs linked to clinical services, promoting team-based care to improve high blood pressure control. www.cdc.gov/dhdsp/pubs/guides/ best-practices/team-based-care.htm. Accessed April 30, 2020.

9. Centers for Disease Control and Prevention. Task Force recommends team-based care for improving blood pressure [press release]. May 15, 2012. www.cdc.gov/media/releases/2012/ p0515_bp_control.html

10. Mitchell P, Wynia M, Golden R, et al. Core principles \& values of effective team-based health care. 2012. Institute of Medicine, Washington, DC.

11. Campbell SM, Hann M, Hacker J, et al. Identifying predictors of high-quality care in English general practice: observational study. BMJ. 2001;323:784-787.

12. Shojania KG, Ranji SR, McDonald KM, et al. Effects of quality improvement strategies for type 2 diabetes on glycemic control: a meta-regression analysis. JAMA. 2006;296:427-440.

13. Walsh JM, McDonald KM, Shojania KG, et al. Quality improvement strategies for hypertension management: a systematic review. Med Care. 2006;44:646-657.

14. Wagner $\mathrm{E}$. The role of patient care teams in chronic disease management. BMJ. 2000;320:560-572.

15. Coleman K, Reid R. Continuous and team-based healing relationships: improving patient care through teams. In: Phillips KE, Weir V, eds. Safety Net Medical Home Initiative Implementation Guide Series. 2nd ed. Seattle, WA: Qualis Health and The MacColl Center for Health Care Innovation at the Group Health Research Institute; 2013.

16. Sinsky CA, Willard-Grace R, Schutzbank AM, et al. In search of joy in practice: a report of 23 high-functioning primary care practices. Ann Fam Med. 2013;11:272-278

17. Howard J, Etz RS, Crocker JB, et al. Maximizing the patient-centered medical home (PCMH) by choosing words wisely. J Am Board Fam Med. 2016;29:248-253. 
18. Solberg LI, Crain AL, Tillema JO, et al. Challenges of medical home transformation reported by 118 patient-centered medical home (PCMH) leaders. J Am Board Fam Med. 2014;27: 449-457.

19. Crabtree BF, Chase SM, Wise CG, et al. Evaluation of patient centered medical home practice transformation initiatives. Med Care. 2011;49:10-16.

20. Carter BL. Blood pressure control-implementing a team approach. US Cardiol. 2011;8:108-113.

21. Carter BL, Rogers M, Daly J, et al. The potency of team-based care interventions for hypertension: a meta-analysis. Arch Intern Med. 2009;169:1748-1755.

22. Proia KK, Thota AB, Njie GJ, et al. Team-based care and improved blood pressure control: a community guide systematic review. Am J Prev Med. 2014;47:86-99.

23. Santschi V, Chiolero A, Colosimo AL, et al. Improving blood pressure control through pharmacist interventions: a meta-analysis of randomized controlled trials. J Am Heart Assoc. 2014;3:e000718.

24. Shaw RJ, McDuffie JR, Hendrix CC, et al. Effects of nurse-managed protocols in the outpatient management of adults with chronic conditions: a systematic review and meta-analysis. Ann Intern Med. 2014;161:113-121.

25. Carter BL, Coffey CS, Ardery G, et al. Cluster-randomized trial of a physician/pharmacist collaborative model to improve blood pressure control. Circ Cardiovasc Qual Outcomes. 2015;8: 235-243

26. Carter BL, Bosworth HB, Green BB. The hypertension team: the role of the pharmacist, nurse and teamwork in hypertension therapy. J Clin Hypertens. 2012;14:51-65.

27. Kennelty KA, Polgreen LA, Carter BL. Team-based care with pharmacists to improve blood pressure: a review of recent literature. Curr Hypertens Rep. 2018;20:1.

28. Brownstein JN, Chowdhury FM, Norris SL, et al. Effectiveness of community health workers in the care of people with hypertension. Am J Prev Med. 2007;32:435-447.

29. Derington $\mathrm{CG}$, King JB, Bryant KB, et al. Cost-effectiveness and challenges of implementing intensive blood pressure goals and team-based care. Curr Hypertens Rep. 2019;21:91.

30. Mills KT, Obst KM, Shen W, et al. Comparative effectiveness of implementation strategies for blood pressure control in hypertensive patients: a systematic review and meta-analysis. Ann Intern Med. 2018;168:110-120.

31. Clark CE, Smith LFP, Taylor RS, et al. Nurse led interventions to improve control of blood pressure in people with hypertension: systematic review and meta-analysis. BMJ. 2010;341:c3995.

32. Santschi V, Chiolero A, Burnand B, et al. Impact of pharmacist care in the management of cardiovascular disease risk factors: a systematic review and meta-analysis of randomized trials. Arch Intern Med. 2011;171:1441-1453.

33. Dennison Himmelfarb CR, Commodore-Mensah $\mathrm{Y}$, Hill MN. Expanding the role of nurses to improve hypertension care and control globally. Ann Glob Health. 2016;82:243-253.

34. Clark CE, Smith LFP, Taylor RS, Campbell JL. Nurse-led interventions used to improve control of high blood pressure in people with diabetes: a systematic review and meta-analysis. DiabetMed. 2011;28:250-261.

35. Zhu X, Wong FKY, Wu CLH. Development and evaluation of a nurse-led hypertension management model: A randomized controlled trial. Int J Nurs Stud. 2018;77:171-178.

36. Spruill TM, Williams O, Teresi JA, et al. Comparative effectiveness of home blood pressure telemonitoring (HBPTM) plus nurse case management versus HBPTM alone among Black and Hispanic stroke survivors: study protocol for a randomized controlled trial. Trials. 2015;16:97.

37. Ogedegbe G. Comparative effectiveness of home BP telemonitoring plus nurse case management (HBPTM+NCM) versus HBPTM alone on systolic BP (SBP) reduction among minority stroke survivors. International Stroke Conference 2020; February 19-21, 2020; Los Angeles, CA. Abstract LB19.

38. Dunn SP, Birtcher KK, Beavers CJ, et al. The role of the clinical pharmacist in the care of patients with cardiovascular disease. J Am Coll Cardiol. 2015;66:2129-2139.

39. Santschi V, Chiolero A, Paradis G et al. Pharmacist interventions to improve cardiovascular disease risk factors in diabetes: a systematic review and meta-analysis of randomized controlled trials. Diabetes Care. 2012;35:2706-2717.

40. Anderegg MD, Gums TH, Uribe L, et al. Pharmacist intervention for blood pressure control in patients with diabetes and/or chronic kidney disease. Pharmacotherapy. 2018;38:309-318.

41. Anderegg MD, Gums TH, Uribe $L$ et al. Physician-pharmacist collaborative management: narrowing the socioeconomic blood pressure gap. Hypertension. 2016;68:1314-1320.

42. Sisson EM, Dixon DL, Kildow DC, et al. Effectiveness of a pharmacist-physician team-based collaboration to improve long-term blood pressure control at an inner-city safety-net clinic. Pharmacotherapy. 2016;36:342-347.

43. Dixon DL, Sisson EM, Parod ED, et al. Pharmacist-physician collaborative care model and time to goal blood pressure in the uninsured population. J Clin Hypertens (Greenwich). 2018;20:88-95.

44. Dixon DL, Parod ED, Sisson EM et al. Impact of a pharmacistphysician collaborative care model on time-in-therapeutic blood pressure range in patients with hypertension. J Am Coll Clin Pharm. 2020;3:404-409.

45. Tsuyuki RT, Houle SK, Charrois TL, et al. Randomized trial of the effect of pharmacist prescribing on improving blood pressure in the community: the Alberta Clinical Trial in Optimizing Hypertension (RXACTION). Circulation. 2015;132:93-100.

46. Tsuyuki RT, Al Hamarneh YN, Jones CA, et al. The effectiveness of pharmacist interventions on cardiovascular risk: The Multicenter Randomized Controlled RxEACH trial. J Am Coll Cardiol. 2016;67:2846-2854.

47. Victor RG, Lynch $\mathrm{K}$, Li N, et al. A cluster-randomized trial of blood-pressure reduction in black barbershops. N Engl J Med. 2018;378:1291-1301

48. Victor RG, Blyler CA, Li N et al. Sustainability of blood pressure reduction in black barbershops. Circulation. 2019;139:10-19.

49. Panattoni L, Hurlimann L, Wilson C, et al. Workflow standardization of a novel team care model to improve chronic care: a quasi-experimental study. BMC Health Serv Res. 2017;17:286.

50. Chang AR, Bonaparte $\mathrm{H}$, Yule C. Randomized controlled trial comparing a self-guided vs. dietitian-led approach using webbased tools to lower blood pressure: study design and rationale. International Stroke Conference 2020; February 19-21, 2020; Los Angeles, CA. Abstract P169.

51. Stephen C, Halcomb E, Mcinnes S, et al. Improving blood pressure control in primary care: The ImPress study. Int J Nurs Stud. 2019;95:28-33.

52. He J, Shi X, Lin M. Comparative effectiveness of implementation strategies on cardiovascular risk factor control in patients with diabetes: The D4C cluster randomized trial. International Stroke Conference 2020; February 19-21, 2020; Los Angeles, CA. Abstract 17. 
53. Jafar $\mathrm{TH}$, Gandhi $\mathrm{M}$, de Silva HA, et al. A community-based intervention for managing hypertension in rural South Asia. N Engl J Med. 2020;382:717-726.

54. Centers for Disease Control and Prevention. Promoting team-based care to improve high blood pressure control. www.cdc.gov/dhdsp/ pubs/guides/best-practices/team-based-care.htm. Accessed April 30, 2020.

55. Jacob V, Chattopadhyay SK, Thota AB, et al. Economics of teambased care in controlling blood pressure: a community guide systematic review. Am J Prev Med. 2015;49:772-783.

56. Dehmer SP, Baker-Goering MM, Maciosek MV, et al. Modeled health and economic impact of team-based care for hypertension. Am J Prev Med. 2016;50(5 suppl 1):S34-S44.

57. Zhang D, Wang G, Joo $\mathrm{H}$. A systematic review of economic evidence on community hypertension interventions. Am J Prev Med. 2017;53:S121-S130.

58. Community Preventive Services Task Force. Cardiovascular disease: team-based care to improve blood pressure control. 2011. www.thecommunityguide.org/findings/cardiovasculardisease-team-based-care-improve-blood-pressure-control. Accessed April 30, 2020.

59. Kulchaitanaroaj P, Brooks JM, Ardery G et al. Incremental costs associated with physician and pharmacist collaboration to improve blood pressure control. Pharmacotherapy. 2012;32:772-780.

60. Mason JM, Freemantle N, Gibson JM, New JP. Specialist nurseled clinics to improve control of hypertension and hyperlipidemia in diabetes. Diabetes Care. 2005;28:40-46.

61. Kulchaitanaroaj P, Brooks JM, Chaiyakunapruk $\mathrm{N}$ et al. Costutility analysis of physician-pharmacist collaborative intervention for treating hypertension compared with usual care. J Hypertens. 2017;35:178-187.

62. Lall D, Engel N, Devadasan N, et al. Models of care for chronic conditions in low/middle-income countries: a 'best fit' framework synthesis. BMJ Glob Health. 2018;3:e001077.

63. Bodenheimer T, Chen E, Bennett HD. Confronting the growing burden of chronic disease: can the U.S. health care workforce do the job? Health Aff (Millwood). 2009;28:64-74.

64. Smith M, Bates DW, Bodenheimer T, Cleary PD. Why pharmacists belong in the medical home. Health Aff (Millwood). 2010;29: 906-913.

65. Wright JT, Williamson JD, Whelton PK, et al. A randomized trial of intensive versus standard blood-pressure control. N Engl J Med. 2015;373:2103-2116.

66. Phillips LS, Branch WT, Cook CB, et al. Clinical inertia. Ann Intern Med. 2001;135:825-834.

67. McManus RJ, Mant J, Franssen M, et al. Efficacy of self-monitored blood pressure, with or without telemonitoring, for titration of antihypertensive medication (TASMINH4): an unmasked randomised controlled trial. Lancet. 2018;391:949-959.

68. Tucker KL, Sheppard JP, Stevens R, et al. Self-monitoring of blood pressure in hypertension: a systematic review and individual patient data meta-analysis. PLoS Med. 2017;14: e1002389.

69. Casey DE, Thomas RJ, Bhalla V, et al. 2019 AHAVACC clinical performance and quality measures for adults with high blood pressure: a report of the American College of Cardiology/American Heart Association Task Force on Performance Measures. J Am Coll Cardiol. 2019;74:2661-2706.

\section{CALL FOR MANUSCRIPTS}

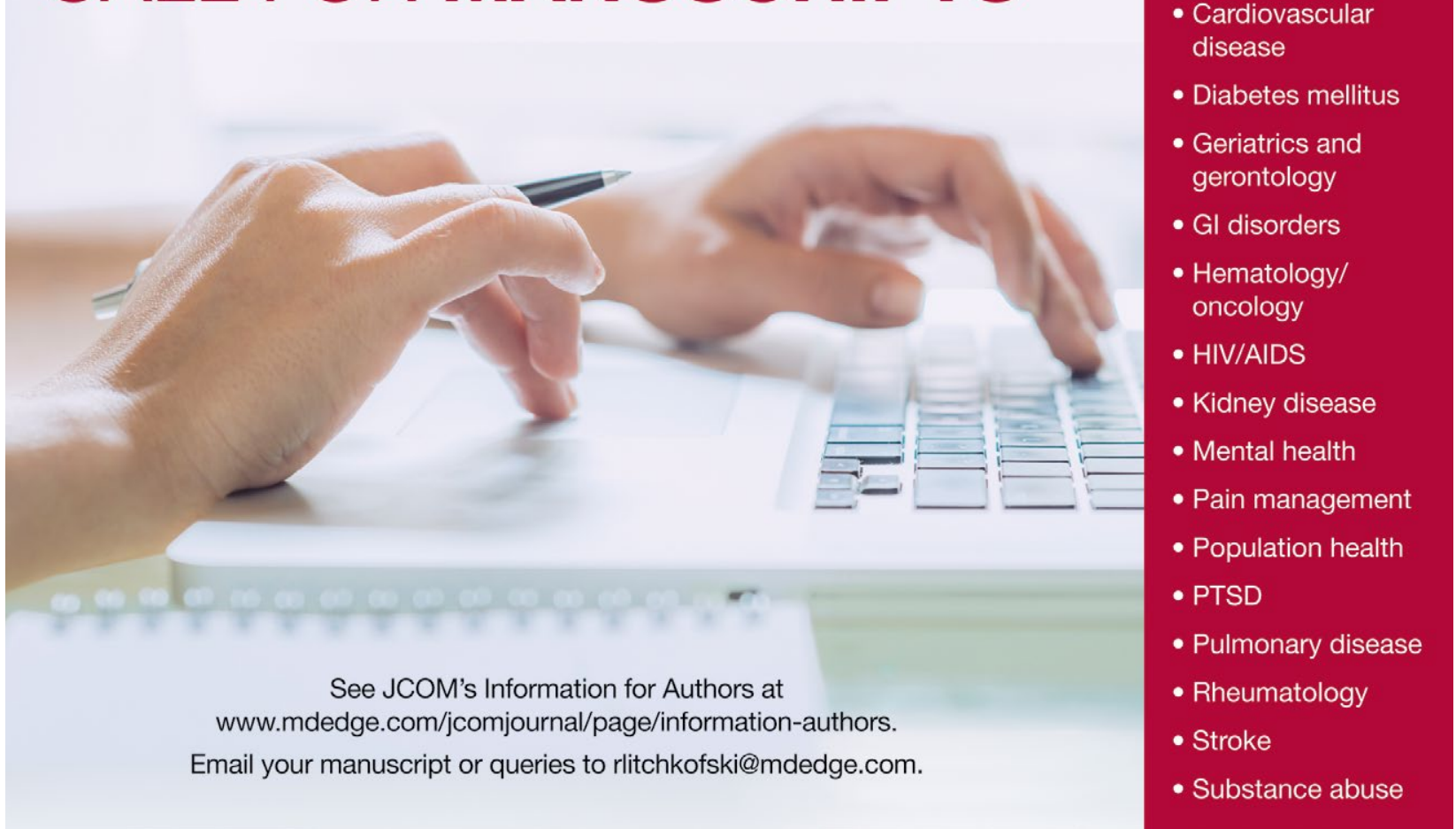

